\title{
P 078 CHALLENGING CHANGE MANAGEMENT: LESSONS FROM THE CAMBRIDGESHIRE ELECTRONIC PALLIATIVE CARE COORDINATION SYSTEM
}

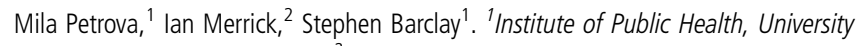
of Cambridge, Cambridge, UK; ${ }^{2}$ independent consultant

10.1136/bmjspcare-2014-000654.119

Background Locality Registers, later EPaCCS (Electronic Palliative Care Coordination Systems), were advanced as key to improving care coordination by the 2008 End of Life Care Strategy. EPaCCS were strongly supported by the National End of Life Care Programme and continue to be a Department of Health priority. In spite of some high-profile successes, most UK localities are struggling to develop their EPaCCS.

Aims This presentation will look into difficulties accompanying the development (ultimately successful) of "Share my Care", the Cambridgeshire EPaCCS. It will discuss "the small, vague, messy and bad things" which the project team had to manage - issues that are commonly ignored by models of change and project management or addressed by solutions that are frequently ineffective.

Methods Analysis of field notes, minutes of team meetings, email communications and interviews with project team members, with reference to social psychology and sociological theory.

Results Key difficulties identified included: precarious balance of different stakeholder groups' visions for the system and the "common" vision; limited interoperability of IT systems; huge challenges of information governance; the dark side of expert knowledge; subtle games of power and need for credit in an ostensibly equal and collaborative playing field; the worm of silent ignorance; the many faces of "good logic" and "highest priority"; the politics of communication, among others.

Conclusions Although the rollout of "Share my Care" across the whole Clinical Commissioning Group is still ahead of us, the developmental project in one Local Commissioning Group has succeeded. This paper, which combines an objective analysis of preceding difficulties with an engaged perspective, self-probing yet self-compassionate, is aimed to motivate colleagues working on EPaCCS. We also aim to enrich models of change and project management in healthcare through attending to some of the nonrational factors involved. 